Slavica

bruxellensia

\section{Slavica bruxellensia}

Revue polyphonique de littérature, culture et histoire

slaves

$8 \mid 2012$

Migration(s) et Exil(s)

\title{
Entretien avec Luba Jurgenson
}

Petra James et Nicolas Litvine

URL : http://journals.openedition.org/slavica/1118

DOI : $10.4000 /$ slavica. 1118

ISSN : 2034-6395

Éditeur

Université libre de Bruxelles - ULB

Référence électronique

Petra James et Nicolas Litvine, «Entretien avec Luba Jurgenson», Slavica bruxellensia [En ligne],

8 | 2012, mis en ligne le 15 juin 2012, consulté le 19 avril 2019. URL : http://journals.openedition.org/ slavica/1118; DOI : 10.4000/slavica.1118

Ce document a été généré automatiquement le 19 avril 2019.

\section{(c) (i) (9)}

Les contenus de Slavica bruxellensia sont mis à disposition selon les termes de la Licence Creative Commons Attribution - Pas d'Utilisation Commerciale - Pas de Modification 3.0 France. 


\title{
Entretien avec Luba Jurgenson
}

\author{
Petra James et Nicolas Litvine
}

\section{Présentation}

1 Luba Jurgenson est née en 1958 à Moscou, ville qu'elle quitta pour la France à l'âge de 17 ans. Elle vit depuis à Paris. En France, elle a suivi un double parcours, celui de l'Université et celui de la littérature. Agrégée de russe (1997) et titulaire d'un doctorat d'Études slaves (2001), elle est actuellement Maître de conférences HDR en littérature russe à l'Université Paris-Sorbonne (Paris IV). Son champ de recherche est celui de la littérature des camps. Elle a publié plusieurs essais dans ce domaine. Elle est également écrivain. Son premier roman parut en 1981. Elle l'a écrit en francais. On lui doit également plusieurs traductions littéraires, donc celle d'ouvrages de Varlam Šalamov ou de Vasilij Grossman par exemple. $\mathrm{Au}$ nombre de ses fonctions, il faut encore ajouter sa co-direction, avec Anne ColdefyFaucard, de la collection Littérature russe " Poustiaki », aux éditions Verdier (Paris).

Recherches en littérature, écriture et traduction sont trois aspects d'une même passion qui a pour point commun la langue. Ou plutôt les langues. La langue francaise, qu'elle utilise au quotidien, dans laquelle elle écrit et traduit, et la langue russe, qui est sa langue maternelle, dans laquelle elle lit et de laquelle elle traduit. C'est ce rapport à la langue, à la distance qui sépare le locuteur du lieu où sa langue est parlée, à la traduction et à la littérature que Luba Jurgenson a bien voulu partager avec nous.

$\mathrm{Au}$ nom de la rédaction de Slavica Bruxellensia nous la remercions pour l'entretien qu'elle nous a accordé.

\section{Entretien}

NiCoLAS LITVINE : Le thème du numéro de Slavica Bruxellensia dans le cadre duquel s'inscrit notre entretien est " exil(s) et migration(s) ». Comment voyez-vous le lien entre ces deux concepts, en particulier dans le monde russe? $Y$ avait-il une différence entre exilés et émigrés à une époque où le départ, dans tous les cas, était perçu comme définitif?

Luba Jurgenson : J'ai tendance à penser - et c'est somme toute assez banal - que l'exil, le sentiment d'exil, s'accompagne de la conscience d'avoir été chassé, banni, expulsé, 
tandis que l'émigration est une démarche volontaire ou du moins consentie. En ce sens, les Russes de la première émigration du $\mathrm{XX}^{\mathrm{e}}$ siècle vivaient leur expérience comme celle d'un exil. Ils avaient été contraints à fuir un pays qu'ils percevaient encore comme étant le leur, après avoir assisté à des bouleversements qui en avaient fait un lieu impossible à vivre, dangereux ; certains d'entre eux avaient perçu le nouveau régime comme une forme d'occupation étrangère. Beaucoup avaient espéré le retour, et certains ont même fini par rentrer - la plupart du temps, pour se retrouver au Goulag ou en relégation. Pour les émigrés de la «troisième vague » à laquelle j'appartiens, les choses se présentaient bien différemment. C'était une émigration à forte composante juive, qui s'est déroulée dans le cadre des accords d'Helsinki, et la plupart des Soviétiques partis dans les années 1970-1980, quels que fussent leurs opinions et leurs projets, ont obtenu l'autorisation de quitter l'URSS grâce à un visa pour Israël. Leur identité se déclinait en bien d'autres termes que celle des premiers émigrés : qu'ils le veuillent ou non, ils étaient des Soviétiques, c'est-à-dire qu'ils étaient nés sous le régime soviétique et avaient été formés par cet État qu'ils percevaient comme répressif et qu'ils voulaient quitter. Cela n'exclut pas, bien sûr, la possibilité d'un déchirement ; ce n'était pas le déchirement de la séparation avec une Russie mythique, c'était l'arrachement à un pays hostile, persécuteur, mais avec lequel ils faisaient souvent corps.

Je laisse de côté la deuxième émigration qui a fui l'URSS dans le sillon de la Seconde Guerre mondiale et que le sentiment de culpabilité et la réprobation générale ont empêché de prendre la parole. À la différence des deux autres vagues, cette population avait un faible pourcentage d'intellectuels, elle n'a donc pas laissé beaucoup de traces écrites.

Mon cas est à la fois typique et singulier, et je me sens plus à l'aise en parlant de moi plutôt qu'au nom d'une collectivité. Typique parce que, moi aussi, je suis partie avec un visa pour Israël, atypique parce que je me suis installée en France, alors que la grande majorité des émigrés allaient aux États-Unis et au Canada. En 1975, au moment où je suis partie, seul un petit pourcentage d'émigrants se rendait en Israël. Dans l'avion qui m'a amenée à Vienne, il s'agissait d'une famille - sur cinq. La France n'accueillait pas les réfugiés d'URSS, je suis donc une exception. Nous avons pu nous établir à Paris parce que nous y avions de la famille. D'autres ont réussi à venir en France parce qu'ils étaient connus en tant que dissidents ou avaient des mérites dans le domaine de la culture, mais l'émigré lambda ne pouvait pas pénétrer sur le territoire français. J'insiste sur ce point parce qu'il existe aujourd'hui une légende selon laquelle les émigrés de la troisième vague, attirés par l'Amérique, auraient «boudé » la France. Ce n'est pas vrai, beaucoup rêvaient de venir en France, mais c'était impossible.

$\mathrm{Au}$ moment de mon départ, je n'avais pas encore dix-sept ans, ce qui a favorisé l'intégration, et en cela aussi je suis atypique. Je quittais un pays que j'avais toujours voulu fuir, élevée dans l'idée que tous les moyens étaient bons pour échapper à l'esclavage et dans la haine absolue du régime. Cette éducation avait fait de moi une personne peu adaptée aux réalités du pays dans lequel je vivais. "Soviétique ", pour moi, voulait dire «hostile». J'avais réussi, par exemple, à ne pas faire partie des jeunesses communistes à une époque où l'adhésion était une sorte de formalité nécessaire à la carrière (les années 1970 étaient celles de la décomposition absolue de l'idéologie, plus personne n'y croyait, mais tout le monde faisait semblant). J'ai donc vécu mon départ comme une libération, une évasion, un miracle. Ce qui n'exclut pas, bien sûr, que ce soit aussi un arrachement. La preuve, c'est que cette expérience est 
finalement devenue fondatrice. Et, bien sûr, en 1975, il s'agissait d'un départ perçu comme définitif, plus que définitif, interplanétaire, un départ pour la planète Mars. La fonctionnaire du service des visas nous avait confisqué nos passeports (nous avions payé d'ailleurs, pour le droit d'en être dépossédées, une bricole de 500 roubles par personne, quatre fois le salaire moyen. L'URSS devait être alors le seul État au monde qui faisait payer le renoncement à la citoyenneté, et non son acquisition). Ironie du sort, elle s'appelait Izrailova (sic!).

Le mot « exil », je l'ai donc découvert en France, lors de débats autour de la culture de l'émigration, auxquels j'ai pu assister ou participer. J'avais alors l'impression (mais j'ai pu nuancer mon jugement, les années passant) que c'était un cliché qui faisait partie des représentations que l'on a des exilés, qu'on l'employait pour faciliter aux Français la perception de notre expérience. Je ne me sentais absolument pas exilée, pas plus qu'un bagnard évadé ne se sent exilé.

Petra JAmes : Vous êtes partie de Moscou à l'âge de dix-sept ans et l'exil fait donc partie de votre expérience personnelle. L'exil est aussi sujet de quelques uns de vos livres, comme Une autre vie. De même, une partie de vos recherches portent sur la question de l'exil. Vous avez traduit des livres écrits par les exilés russes. Le fait d'aborder cette expérience dans des contextes divers aide-il à mieux le saisir?

Je l'ai dit, c'est une expérience fondatrice. Au début, on ne s'en doute pas, on part pour commencer une vie ailleurs. Et peu à peu, on se rend compte que le récit de soi, ce récit que l'on fait pour soi-même de sa vie ailleurs, est déterminé par ce qui était au commencement : le départ. Le fait de pouvoir retourner dans le pays d'où l'on est parti n'efface pas cette coupure, il la ravive même, mais permet aussi de la penser, et autrement qu'en termes de perte. À ce titre, tous mes livres portent sur l'exil - mais de manières différentes. Appréhender l'exil sous ses diverses formes par l'écriture, la recherche, la traduction, fait que cette expérience s'inscrit en genèse des trames du vécu - acquiert un statut presque mythologique, celui d'un mythe personnel. On prend ainsi conscience de la façon dont les mythes tissent notre existence. C'est donc un certain rapport au réel que cette réflexion permanente induit: on perçoit alors très fortement la dimension narrative de son propre vécu. Ce que nous vivons, c'est aussi ce que nous racontons de notre vécu. Bien entendu, cela ne concerne pas que les bilingues exilés, mais le fait de réfléchir sur l'exil accentue ce phénomène. S'interroger sur le sens - y compris le sens des choses, le sens du vécu - c'est construire un récit. Au fondement d'un récit de l'exil, il y a toujours un déboulonnage du sens, une déconstruction des évidences, des données du réel. Peut-être que je dis tout cela parce que j'appartiens à une génération touchée par le post-modernisme, mais il me semble que ce fait générationnel n'aurait pas trouvé à s'exprimer en moi de manière aussi forte s'il n'y avait pas eu l'expérience de l'exil au départ.

N. L. : Peut-on parler d'un exil ou d'une émigration proprement russe/soviétique, particulièrement dans un pays où l'exil et le déracinement ont souvent été une expérience vécue à l'intérieur des frontières?

J'ai déjà répondu en partie à cette question, je risque donc de me répéter un peu. En ce qui me concerne, je me sentais étrangère en vivant en URSS, je refusais de me considérer comme soviétique. Le mode de refus choisi par ma famille était un repli absolu sur soi et sur des valeurs «bourgeoises » fustigées par l'idéologie ambiante. Ma famille a poussé cette forme de résistance passive très loin, jusqu'à l'inadaptation. Je ne sais pas très bien ce que je serais devenue si nous n'avions pas pu émigrer, mon éducation me donnait très peu de chances de m'intégrer à la société soviétique, de faire 
des études supérieures. Je me serais probablement tournée vers la culture clandestine, qui était alors une dimension importante de cette société. Du moins, c'est ainsi que je vois les choses maintenant. La question ne s'est finalement pas posée. Pourtant, d'une certaine manière, j'étais une Soviétique, tous ces problèmes faisaient justement de moi une Soviétique, avec une histoire familiale qui n'eût été possible que dans ce pays. J'avais eu le temps de me frotter à la «culture» de la rue ou plutôt de la cour moscovite, haut lieu de l'éducation. J'ai toujours en moi cette enclave, résidu d'un monde inexistant.

Dans mon cas, le désir d'émigrer partait d'une volonté affirmée de ne pas m'installer dans cette situation d'exil intérieur. Car elle était le fruit d'une totale absence de liberté. La société soviétique était une société où toute forme de non-conformisme se transformait immédiatement en marginalité, laquelle était une forme de délit - le fait de ne pas travailler pour l'État devenait du parasitisme, le commerce privé était de la spéculation, l'« apolitisme " (l'absence d'affiliation à une organisation) pouvait être vu comme une position antisoviétique (ce qu'il était de fait), etc. Je désirais ardemment ne pas vivre dans cette situation toute ma vie, pouvoir décider de mon existence sans que cela n'apparaisse immédiatement comme un choix politique.

Parmi les émigrés de ma vague, tous ne se sentaient pas des exilés de l'intérieur. Et inversement, tous les exilés de l'intérieur n'ont pas émigré - cette position les prédisposait souvent à l'action au sein du pays, ou bien à une sorte d'existence semilégale dont le régime brejnévien permettait de s'accommoder tant bien que mal. Malgré tout, on n'était plus au temps de Staline. Nous jugeons aujourd'hui d'après la minorité qui a laissé une trace dans la culture - mais la grande majorité, dans cette vague, était formée par des gens qui avaient réussi malgré tout à trouver un modus vivendi en URSS. À Vienne, où nous avons séjourné en attendant d'entrer en France, j'ai pu rencontrer des gens qui ne savaient pas très bien pourquoi ils étaient partis. Ou plutôt, ils étaient partis parce que leurs voisins étaient partis, parce que tout le monde partait. Il n'était pas facile d'être juif en URSS, mais cela ne voulait pas dire nécessairement que l'on se sentait en position de rejet par rapport au pays.

N. L. : Dans une interview donnée l'an passé à France-Culture 1, vous évoquiez les conséquences de la désintégration de I'Union Soviétique sur la manière dont vous perceviez votre identité. Est-ce que vous pourriez évoquer le sens que cet événement a eu pour vous? Cela a-t-il changé votre rapport à l'usage de la langue russe dans l'écriture, précédemment ressentie comme la langue des dissidents, une langue qui exprimait un vécu fort, souvent tragique.

Pour l'expliquer, je reviens d'abord à la différence entre exilé et émigré. Une identité d'exilé est pour moi une sorte d'identité négative - on se définit par rapport à un « chez soi » perdu. En revanche, l'émigration est presque une sorte de patrie, un îlot au sein d'un autre pays, une enclave - une diaspora. Je n'avais pas eu de « chez moi » en Union soviétique. Tandis que l'émigration russe en France, c'était une grande maison, un immense territoire culturel dont l'héritage était plus riche et plus facile à assumer, sur le plan esthétique et politique, que celui de la culture soviétique dans son ensemble. Ce n'est pas que je dévalorisais l'héritage soviétique - j'aurais du mal à dire, par exemple, lequel des deux, Vladimir Nabokov ou Andrej Platonov, a compté davantage - mais je ne me sentais pas une héritière légitime de cette culture-là, entre autres parce que je l'avais fuie, j'avais placé ma vie en-dehors de cette histoire. J'ai donc fini par me forger une identité que je pourrais définir comme "russe émigrée », et cette identité m'a permis de m'intégrer en France. Les émigrés «blancs » avaient beaucoup souffert, en 
leur temps, du rejet et de l'indifférence des Français mais, à l'époque où je suis venue, c'était un statut valorisé et confortable. Or, ce « récit de soi » que l'on promène en le croyant collé à la peau peut en réalité changer. Lorsque l'URSS a disparu et qu'a émergé une Russie dans laquelle je ne me reconnaissais pas - et ne me reconnais toujours pas -, lorsque nombre d'émigrés ont noué des liens avec cette nouvelle Russie, réalisant en quelque sorte leur rêve de retour, et que la notion même d'émigration a perdu son sens (on pouvait désormais rentrer en Russie sans encourir de persécutions politiques), mon habit d'émigrée s'est effiloché très vite. C'est alors que d'autres facettes identitaires sont revenues dans mon champ visuel. Je peux l'illustrer par l'exemple de mes livres. Toute notion d'ancrage géographique ou historique est absente du premier ${ }^{2}$, mais alors, radicalement. Quand il est paru (en 1981), les journalistes ont cherché à me faire dire que ce livre exprimait la nostalgie de la Russie, et j'ai résisté farouchement. Le deuxième $^{3}$ a pour personnage principal un objet de musée, je crois que cela exprime assez bien mon rapport au pays d'origine. Ce livre a eu un chemin difficile, les éditeurs n'en voulaient pas, et, cherchant à le rendre accessible, j'ai peu à peu fait de ce musée une sorte de musée d'arts et traditions populaires russes, mais c'était encore une Russie de musée. Dans le suivant, Une Autre Vie $e^{4}$, j'ai raconté les détails de mon départ d'URSS je suis contente de l'avoir fait, car ces détails, on finit par les oublier, et aujourd'hui, c'est déjà de l'histoire. Puis, il y a eu un roman sur les milieux artistiques de Moscou, un roman que j'avais commencé à écrire immédiatement après l'arrivée en France, mais que je n'ai pu l'achever qu'au moment de la Perestroïka. C'est ainsi que la Russie (une Russie fortement mythifiée) est venue à moi peu à peu à travers les livres. Dans Le Soldat de papier ${ }^{5}$, ce roman, donc, l'imagination et la réalité se sont rejointes - car au moment où je le terminais, j'ai pu retourner à Moscou pour la première fois et revoir les gens dont j'avais fait mes personnages - sans leur en parler, bien sûr. Comme j'écrivais en français, ils n'avaient aucune chance de s'en rendre compte. C'était une expérience de déguisement vertigineuse. Le livre a paru en 1989, à peu près au moment de la chute du mur de Berlin. Je n'arrivais pas à croire que ce régime avait pris fin. J'ai appris la chute du Mur sur un plateau de télévision, qui était situé sur un vrai plateau, en montagne, dans le Sud-Ouest de la France, et je me souviens d'avoir dit - pour " frimer », mais pas seulement: «Quel mur? » Je croyais que c'était encore une ruse du régime, je ne me rendais pas compte que cet événement allait changer non seulement les frontières de l'Europe, mais aussi ma géographie intérieure. Car en fait, Le Soldat de papier était mon dernier livre " russe ». La chute du Mur m'a emmenée vers l'Allemagne, et depuis, sont nés trois livres sur des thèmes allemands - une Allemagne inventée et mythique, bien sûr - dont le dernier vient de sortir ${ }^{6}$. Situer leur action en Allemagne m'a permis d'intégrer une facette de mon identité que j'avais mise entre parenthèses, la judéité, qui m'a été révélée en partie, paradoxalement, grâce à la chute du Mur, il y a un mur qui est tombé en moi aussi.

Depuis, l'objet principal de mes livres, c'est la langue. Un des objectifs qui s'est dessiné peu à peu dans l'écriture, c'est de créer des langues différentes au sein du français et de jouer avec ces langues, de vivre la situation de plurilinguisme au sein du texte. Mon dernier livre, Trois contes allemands, porte essentiellement sur la langue. La préoccupation babélienne s'est imposée comme mode d'appréhension du rapport entre l'individu et l'histoire : la question de l'histoire dans les vies individuelles, dans ma vie, est abordée à travers diverses aventures langagières.

J'ai dit, il est vrai, que dans mon choix du français comme langue d'écriture, a pesé la violence de l'histoire russe et soviétique, qui avait trouvé à s'exprimer dans la langue et 
qui l'avait en partie façonnée. Il faut y voir un rapport à la langue de quelqu'un de très jeune, qui commence à écrire et qui, avec cette acuité et intransigeance de la jeunesse, se pose la question de la responsabilité de l'écrivain devant la langue, le langage. En arrivant en Occident, j'ai découvert l'ampleur des répressions et des crimes de l'État soviétique. Une avalanche de livres, à la fois de dissidents et d'émigrés des époques précédentes. Je savais que j'avais fui un État criminel, mais j'ignorais le détail et la complexité de cette histoire. Cela m'a écrasée. Comment trouver sa place d'écrivain face à tout cela? J'avais choisi de fuir, non de lutter. Depuis longtemps, il ne se passe pas de jour sans que je me réjouisse d'avoir fait ce choix. Mais à l'époque, à dix-sept ans, j'ai eu pendant quelques mois l'impression d'avoir fait fausse route, d'avoir manqué l'occasion d'être dans l'action et non dans la parole. Puis, j'ai assumé ce choix en optant pour le français. Il fallait trouver une langue en adéquation avec cette position, et cette langue, c'était le français. Car l'histoire, c'est aussi la langue. Comment déserter l'histoire soviétique? (Qui, soit dit en passant, est devenue plus tard pour moi un objet d'étude.) En désertant la langue dans laquelle elle s'écrit.

P. J. : La problématique de l'exil est étroitement liée à celle de l'identité. Vous même, vous êtes à la fois écrivaine, traductrice, chercheuse, enseignante... Comment ces différentes identités se combinent-elles en vous?

Ces identités ne se sont pas construites en même temps, elles sont venues se superposer les unes aux autres, avec une certaine logique. D'abord, il y a eu celle d'écrivain, j'ai commencé par des textes de fiction. Ensuite, est venue l'envie de traduire, sans doute pour maintenir un contact avec la littérature russe que j'avais quittée en tant qu'écrivain, et aussi, pour « rester un peu sur la route », donner un mouvement à mon univers littéraire, ne pas me sentir "arrivée", mais pouvoir faire l'expérience du retour - par la langue. Le rapport à l'enseignement et à la recherche a été bien plus complexe. Au moment où je devais passer l'Agrégation, en 1988, je bifurquais vers une activité d'écrivain et de traductrice à part entière, pour ne revenir vers l'université qu'en 1996. Je ne croyais pas pleinement à ma légitimité à transmettre des savoirs. Il a fallu un long chemin pour comprendre que l'on ne transmettait pas des savoirs comme des entités figées, mais des outils, des capacités de penser, des aptitudes critiques. Ce qui a déclenché le désir d'intégrer une communauté de chercheurs et d'enseigner, c'est encore l'expérience de l'écriture et de la traduction. Pour un de mes livres, Éducation nocturne, j'avais fait beaucoup de recherche sur la Shoah, j'avais vécu à Berlin, et il $\mathrm{y}$ avait le besoin de continuer de réfléchir sur ces questions. J'étais parmi les traducteurs du Livre noir ${ }^{7}$ de Vassilij S. Grossman et ILja G. Ėrenburg, et là, tout d'un coup, je me suis sentie dépassée par ce que j'étais en train de traduire. C'était insupportable. J'ai compris alors qu'on ne pouvait pas travailler seul sur les violences historiques, qu'il fallait intégrer une communauté de chercheurs. C'était un sentiment très fort d'isolement, d'absurde, je me suis sentie en danger, et puis, cela n'avait aucun sens de réfléchir sur la Shoah et le Goulag dans mon coin, ce sont des faits qui questionnent toute la culture européenne, il était nécessaire d'inscrire cette réflexion dans une réflexion commune. Cela a été comme une révélation: je me suis immédiatement inscrite au concours de l'Agrégation, et j'ai enchaîné avec la thèse.

Bien évidemment, ces identités interfèrent. Le fait d'avoir travaillé sur le témoignage littéraire a bouleversé mon rapport à l'écriture. Il y a dans ma vie, par exemple, un « avant » et un « après » Šalamov. On ne peut écrire de la fiction innocemment après avoir lu Kolymskie rasskazy (Les Récits de la Kolyma) ${ }^{8}$. Je ne dis pas que j'écrivais tout à fait « innocemment », mais j'ai quand même écrit deux romans qui sont, somme toute, 
de facture assez traditionnelle, en ce sens qu'on y trouve une intrigue, une succession de chapitres, etc. Je n'ai plus écrit de romans comme ça. Le jeu avec la forme est devenu beaucoup plus important. Boutique de vie commence comme un recueil de nouvelles pour se transformer en roman. Et le dernier, Trois contes allemands, présente trois histoires différentes, et c'est pourtant un roman.

Le fait de côtoyer les textes des témoins a aussi changé le rapport à ce que l'on donne à voir dans la narration. Par exemple, dans Éducation nocturne, il y avait des scènes qui se déroulaient à Auschwitz. Dans une version ultérieure, en russe, je les ai enlevées ${ }^{9}$. Je ne comprenais même pas comment j'avais pu les écrire - il était évident qu'on ne pouvait pas montrer le camp, qu'il ne fallait pas le faire, mais c'était évident pour la chercheuse qui avait suivi toutes les polémiques autour du «montrer ». À l'époque où j'écrivais la première version de ce livre, la Shoah n'était pas un thème aussi présent dans l'espace mémoriel et académique qu'il l'est à présent. Aujourd'hui, franchement, il est impossible de montrer un camp au premier degré. Enfin, ce n'était pas tout à fait au premier degré dans la première version non plus, il s'agissait d'une déconstruction aussi, d'un livre fondamentalement ironique, mais cette ironie n'était peut-être pas perceptible à chaque instant. Dans la deuxième version, l'ironie est montée d'un cran.

Par ailleurs, les recherches sur la mémoire ont influé sur mon écriture en ceci : je n'écris pas sur l'histoire, mais uniquement sur des représentations de l'histoire, des constructions, des contorsions de la mémoire.

N. L. : Vous avez un jour indiqué qu'en 1990, vous parliez encore du français comme d'une langue étrangère. Aujourd'hui, le bilinguisme apparaît comme un élément central de la manière dont vous percevez votre identité, comment interprétez-vous cette évolution ?10

J'ai été très surprise en découvrant ce texte que j'avais écrit, je me suis même demandé si j'avais été sincère, si ce n'était pas juste une pirouette verbale. Mais j'ai constaté aussi que dans ce texte autobiographique, il y avait encore des éléments de mon expérience soviétique que je n'arrivais pas à raconter. En particulier, un épisode survenu pendant que nous faisions des démarches pour quitter l'URSS. Il fallait dire partout que nous allions en Israël et ne pas parler de la France. Or, le directeur de mon lycée, qui refusait de me délivrer un papier dont j'avais absolument besoin pour constituer le dossier de demande de départ, a réussi, après m'avoir "cuisinée » au cours d'entretiens qui se répétaient de jour en jour, à me faire plus ou moins avouer que notre destination était la France. J'ai raconté cet épisode dans Une Autre Vie, mais pas jusqu'au bout, et dans ce texte de 1990, je passais sous silence certains aspects de cet épisode. J'en avais terriblement honte, car à l'époque, en 1975, j'avais eu l'impression d'avoir trahi ma famille et craint d'avoir probablement compromis notre départ. En réalité, cela fut sans conséquences, mais je me projetais imaginairement dans l'époque stalinienne et je vivais ces entretiens, somme toute assez malveillants et retors, comme un véritable interrogatoire dont l'enjeu était la vie et la liberté. En 1990, quinze ans plus tard, cette honte était, manifestement, encore là. Aujourd'hui, je peux en parler sans la moindre gêne, peut-être justement parce qu'il y a eu ce passage du français « langue étrangère " au français «langue consubstantielle». La continuité avec mon passé soviétique est maintenant de l'ordre du récit - je peux en parler comme si je parlais de quelqu'un d'autre. Lorsqu'on me demande aujourd'hui si le français est une langue acquise, je dis non - ce n'est pas une langue acquise. Pourtant, il y a eu un moment dans ma vie où j'apprenais le français, mais par la suite, le français est devenu ma langue, qui a le pouvoir de raconter rétrospectivement mon histoire - une histoire bien différente de celle que je pouvais raconter auparavant. Je l'ai adopté assez vite (au bout de deux ans 
en France), mais ce passage est apparu, après coup, une naissance - et donc la langue du passage comme une langue natale.

Dans les années qui ont suivi la chute du Mur et la dissolution de l'Union soviétique, j'ai participé à de nombreuses rencontres, en France et en Allemagne, sur le bilinguisme, sur le fait d'écrire dans deux langues. J'avais alors l'impression que le statut de bilingue, d'écrivain bilingue, le statut d'entre-deux en général était alors valorisé, ou du moins reconnu, et suscitait de l'intérêt dans cette nouvelle Europe dont les frontières bougeaient. Cette donnée m'a certainement aidée à prendre conscience de la dimension «existentielle» du bilinguisme comme mode d'être particulier, dans lequel je me reconnaissais désormais. Puis, progressivement, on a assisté à la montée des communautarismes, et ce statut particulier, presque institutionnalisé dans sa marginalité, a été, pour autant que je puisse en juger, perçu comme l'expression d'un manque, d'une douleur, d'une perte irrémédiable. J'ai vu passer des publications où l'exil était traité comme un traumatisme presque sans distinction de cas. Dans une Europe désormais hantée par la question identitaire - obsession des communautés qui se sentent en danger - le bilingue, avec son identité complexe apparaît d'une certaine manière comme une menace (et ce, à l'heure où l'expérience du bilinguisme est partagée par tant de monde!), comme une présence subversive, et on cherche à le comprendre à travers des notions comme l'arrachement, le deuil, etc. Le mot " patrie » a refait surface dans les discours sur l'exil. J'ai alors éprouvé le besoin de penser plus à fond cette question de bilinguisme, d'affirmer, puisque en tant que bilingue j'en ai l'expérience et pas seulement la conviction, que l'identité n'est pas une donnée intangible, que c'est une construction.

N. L. : Ayant appris et usé quotidiennement des deux langues, le russe et le français à des âges et dans des contextes différents, ressentez-vous une manière différente de percevoir les choses dans les deux langues (et dans l'affirmative, comment gérez-vous cette différence dans le travail de traduction)?

Si je voulais trouver une formule simple, je dirais qu'il y a une langue sujet et une langue objet. Le français, la langue dans laquelle j'écris, vers laquelle je traduis est la langue dans laquelle je suis active. Et le russe, la langue dont je traduis, sur laquelle je réfléchis. Le russe est la langue de l'enfance, et d'une certaine manière, dans cette langue j'aurai toujours dix-sept ans. Je ne l'ai pas travaillée dans l'écriture, c'est pourquoi, il y a un clivage entre l'oral et l'écrit. Je peux faire cours en russe, écrire un article, mais je ne peux pas écrire un texte littéraire, ou bien seulement des bribes, des fragments, surtout poétiques, peut-être parce que je régresse alors vers cet âge où tout le monde écrit des poèmes. Les Russes qui me connaissent ont beaucoup de mal à me croire quand je raconte cela, car à l'oral, cette incapacité ne se perçoit pas. En réalité, les choses sont plus complexes. Mon russe nourrit mon français - il y vit clandestinement tout comme les références à la littérature russe. Ce qui serait perçu comme une dépendance à l'égard des auteurs qui m'ont marquée ne l'est plus dans la mesure où j'écris en français. Je n'ai pas eu à me débarrasser des influences, problème de tous les jeunes auteurs, je les ai cultivées «à la barbe » de tous. Le bilinguisme a changé le rapport à ce qui est caché, à ce que l'on fait circuler en contrebande dans un texte littéraire. Je suis extrêmement attentive à cette dimension de la littérature, aux cryptages, aux polyphonies, à l'intertextualité, ce qui a déterminé en grande partie les axes de ma recherche. Ce n'est pas un hasard si je travaille sur les silences, les non-dits, les contenus cachés des textes. Le bilinguisme permet aussi de s'observer, d'investir la relation sujet-objet comme une relation à soi. 
La traduction est devenue possible à partir du moment où j'ai eu envie que le français devienne ma langue - on ne traduit que vers sa langue. Mais cette position particulière de traductrice a sans doute influé sur ma façon de travailler. Je suis préoccupée, peutêtre davantage que mes collègues français de naissance, par la fluidité du texte, je cherche toujours à ce qu'il donne l'impression d'être écrit en français, cet impératif prime dans mes choix. Cela dit, en traduisant, je me mets au service du texte, je fais de moi un réceptacle pour le texte d'autrui. Je ne relis pas mes traductions une fois qu'elles sont publiées, sauf quand j'ai l'occasion de les reprendre pour une nouvelle édition, mais j'imagine que ma façon de traduire évolue aussi, change, se travaille. Il y a un bonheur à se laisser traverser par des textes, à vivre le travail de la traduction comme une expérience presque physique, c'est une activité beaucoup moins autoréflexive que celle de l'écriture ou de la recherche. Je ne me demande pas trop « où j'en suis » quand je traduis, pas plus qu'un ver à soie ne se demande ce qu'il est en train de faire (que les vers à soie me corrigent si je me trompe). De manière générale, le bilinguisme est aussi une expérience physique, qui s'inscrit dans le corps, et donc, sujette au changement.

P. J. : En tant que traductrice vous faites un aller-retour constant entre le russe et le français, vous traduisez dans les deux sens. Comment était pour vous le processus de l'acceptation de la nouvelle langue de communication après votre départ de l'Union soviétique? Est-ce que vous êtes passée par une phase de refus du russe pour construire votre nouvelle identité?

Je ne me souviens pas d'une telle phase de refus. En revanche, je me souviens de la jubilation à m'approprier le français, à sentir émerger de nouveaux sens, de nouveaux rapports entre les sons et le sens. Je m'amusais à utiliser des mots que je ne connaissais pas. Par exemple, dans mon premier livre, je trouve cette phrase : «le sommeil dont je dormais était lisse, astringent, concave ». C'est étrange et cela ne veut pas dire grandchose, je n'écrirais pas cela aujourd'hui. Mais ces mots me plaisaient, je les découvrais, je saisissais leur sens « au toucher » plus que par l'ouïe. Évidemment, quand on fait de la traduction, on n'a pas le droit de faire ça - mais d'une certaine manière, l'émerveillement devant les mots, y compris leur être physique, ne m'a jamais quittée.

En fait, il s'agit pour moi uniquement d'auto-traduction vers le russe, et cette expérience est relativement récente, elle est liée à mon travail de traduction vers le français. J'avais très envie de voir publié en russe un de mes livres, mais l'occasion ne se présentait pas. En 2004, Leonid Giršovič que je traduis et publie dans ma collection ${ }^{11}$, m'a proposé de lui faire lire une page d'un de mes livres, n'importe laquelle. J'ai traduit un passage d'Éducation nocturne, c'était une traduction presque littérale, un premier jet. Il l'a retravaillée ensuite. Le travail que le traducteur fait seul - et moi, je le fais toujours en deux étapes - a été ici réalisé à deux. La lecture de cette page m'a bouleversée. J'avais l'impression de me lire pour la première fois. Quand on écrit dans une langue qui n'est pas votre langue maternelle, il y a comme une sorte d'écran entre soi-même et ce qu'on écrit (un écran protecteur, salutaire, mais écran tout de même). Et voilà qu'il était tombé. C'était une expérience absolument inédite de retour dans sa langue. Du coup, nous avons entrepris de traduire l'ensemble du livre. Nous avions déjà une longue expérience de travail en commun, d'exégèse en commun, car les textes de Giršovič, il faut beaucoup les commenter pour arriver à les traduire. Le livre a paru à Moscou en 2009 et en ce moment, nous traduisons Trois contes allemands, mon dernier roman. J'ai écrit un petit texte sur cette expérience de traduction à quatre mains, «Traduire ensemble », disponible sur le site des éditions Verdier. 
P. J.: Est-ce que dans la prose contemporaine russe vous ressentez un changement important de style et de vocabulaire?

Le syndrome de l'émigré qui a l'impression que la langue se dégrade, que la qualité du style baisse, ne m'a pas épargnée, même si je lutte contre la tentation du "purisme ». Bien sûr, dans la production de masse, entre l'époque soviétique et aujourd'hui, c'est le jour et la nuit, sur tous les plans : éthique, esthétique, culturel. Mais au fond, cette production de masse ne m'a jamais intéressée, ni en russe ni en français. Les auteurs russes contemporains que je cherche à faire connaitre en France sont de grands stylistes. Il est vrai que je n'en trouve pas tous les jours. Dans notre collection chez Verdier, il y a deux auteurs contemporains, Leonid Giršovič et Vladimir Sorokin ${ }^{12}$. Et j'ai découvert récemment un jeune auteur, Sergej Lebedev, que je vais publier, il écrit merveilleusement bien. Il arrive que des personnes pour lesquelles j'ai de l'estime me recommandent un livre - et voilà qu'il me tombe des mains. Ce n'est pas seulement une affaire de style, c'est un certain manque de complexité, des textes taillés à la hache. En cela, la littérature russe contemporaine s'aligne sur toutes les autres. On n'y peut rien. J'ai souvent l'impression que c'est un orchestre où il ne reste que des percussions - tous les autres instruments ont disparu. Le lecteur n'est pas devenu plus bête pour autant, mais il semble vouloir du bruit, beaucoup de bruit, et n'a plus la disponibilité de prêter l'oreille à des sons plus subtils. Ou peut-être que les écrivains fabriquent ce lecteur. En tout cas, il y a des écrivains qui ont su s'adapter à cette demande sans rien perdre de leur complexité, comme Sorokin, et d'autres non.

En disant tout cela, je suis bien consciente de raisonner en personne de ma génération. Les écrivains de l'Âge d'Argent, ceux qui sont un modèle pour moi, étaient perçus par ceux qui les ont précédés comme des démolisseurs. Lisons ce que Tolstoj dit du symbolisme! Comme il fustige Baudelaire! Et il suffit de se rappeler la réaction de Gide à la lecture de Proust. Peut-être que parmi les textes qui me semblent primitifs et grossiers, il y a des œuvres géniales, que je suis incapable de reconnaître.

P. J. : Votre travail de traduction est systématique et vaste. Quels sont les critères selon lesquels vous choisissez les auteurs que vous souhaitez traduire?

Il y a les textes que je choisis, que je porte, dont je suis responsable (par exemple, les textes que je traduis pour la collection que je co-dirige avec Anne Coldefy aux éditions Verdier) et ceux que l'on me propose. Le critère, c'est de se sentir en adéquation avec le texte. Je sens vraiment que certaines de mes traductions sont mieux réussies que d'autres. Si je regarde la liste des textes traduits, j'y trouve essentiellement de la littérature de témoignage - Varlam T. Šalamov, Julius Margolin ${ }^{13}$, Jurij Čirkov ${ }^{14}$, Le Livre noir, tout récemment, le Journal d'un gardien du Goulag ${ }^{15}$, etc., des textes de l'Âge d'Argent ${ }^{16}$ : Marina I. Cvetaeva ${ }^{17}$, Nina N. Berberova ${ }^{18}$, Mikail Zenkevič et aussi, de la littérature contemporaine (Friedrich Gorenstein ${ }^{19}$, Leonid Giršovič ${ }^{20}$, Anatolij V. Korolëv $\mathrm{v}^{21}$ ). Ce sont là les trois principales orientations. Quand j'ai commencé à traduire, c'était surtout pour faire connaître la littérature contemporaine qui dans les années 1970 comprenait de très grands textes. Mais je me suis formée en traduisant des classiques - même si aujourd'hui je ne le fais plus - notamment, Oblomov, grâce à la confiance très généreuse de Jacques Catteau qui m'avait donné l'occasion de réaliser la traduction de ce texte pour sa collection à l'Âge d'Homme, alors que je débutais ${ }^{22}$. 


\section{NOTES}

1. Cette interview, réalisée le 16 janvier 2011, est disponible en streaming sur le site de FranceCulture à l'adresse http://www.franceculture.fr/emission-tire-ta-langue-10-11-lubajurgenson-2011-01-16.

2. Jurgenson L., Avoir sommeil, Gallimard, 1981, Paris, 133 p.

3. Jurgenson L., L'Autre, Albin Michel, Paris, 1984, 176 p.

4. Jurgenson L., Une Autre vie, Lieu commun, Paris, 1986, 189 p.

5. Jurgenson L., Le Soldat de papier, Albin Michel, Paris, 1989, 215 p.

6. Jurgenson L. : Éducation nocturne, Albin Michel, Paris, 1994, 390 p. ; Boutique de vie, Actes Sud, Paris, 2002, 208 p. ; Trois contes allemands, Pierre-Guillaume de Roux éd., Paris, 2012, 366 p.

7. Le Livre noir (textes et témoignages réunis par Ėrenburg [Ehrenbourg] I. \& Grossman V.), Parfenov M. (dir.), traduit du russe par Yves Gauthier, Luba Jurgenson, Michèle Kahn, Paul Lequesne \& Carole Moroz, Solin-Actes Sud, Arles, 1995, 1134 p.

8. Les Kolymskie rasskazy (Les Récits de la Kolyma) ont été écrits par Varlam T. Šalamov entre 1954 et 1973. La première parution en russe date de 1978; la première parution en URSS date de 1987. Nouvelle édition francaise : Récits de la Kolyma, traduit du russe par Catherine Fournier, Sophie Benech \& Luba Jurgenson, préface de L. Jurgenson (maître d'œuvre), postface de M. Heller, Éditions Verdier, 2003, 1536 p.

9. Voir plus bas.

10. Entretien du 23 mai avec Claude Mouchard dans le cadre des «Entretiens de Po\&sie ». Un compte-rendu de cet entretien est disponible à l'adresse: http://poezibao.typepad.com/ poezibao/2011/05/une-rencontre-avec-luba-jurgenson.html, un extrait (mais pas celui auquel il est ici fait référence) peut également être visionné à l'adresse: http:// lesentretiensdepoesie.blogspot.be/2011/05/luba-jurgenson.html.

11. Il s'agit de la collection Littérature russe «Poustiaki », dirigée par Anne Coldefy-Faucard et Luba Jurgenson, aux éditions Verdier (Paris). (NdlR)

12. Sorokine [Sorokin] Vl. : Roman, traduit du russe par Anne Coldefy-Faucard, Verdier, Coll. «Poustiaki », 2010, 608 p.; La Tourmente, traduit du russe par Anne Coldefy-Faucard, Verdier, Coll. « Poustiaki », 2011, 192 p.

13. Margolin J., Voyage au pays des Ze-Ka, traduction du russe par Nina Berberova et Mina Journot, révisée et complétée par Luba Jurgenson, Le Bruit du temps, Paris, 2010, 781 p.

14. Tchirkov I. [Čirkov Ju.], C'était ainsi... : Un adolescent au Goulag, traduit du russe par Luba Jurgenson, Editions des Syrtes, Coll. « Littérature étrangère ", Paris, 2009, 367 p.

15. Tchistiakov I., Journal d'un gardien du Goulag, traduit du russe par Luba Jurgenson, préface de Scherbakova I., Denoël, 2012, 286 p.

16. Deux dernières décennies $d u X X^{\mathrm{e}}$ siècle et années précédant la révolution bolchévique d'octobre 1917. (NdlR)

17. Tsvetaeva [Cvetaeva] M., Carnets, publiés sous la direction de Jurgenson L., traduits du russe par Éveline Amoursky et Nadine Dubourvieux, préface de Bérenger C., postface de Lossky V., Éditions des Syrtes, Paris, 2008, 1136 p.; Boris Pasternak - Marina Tsvetaeva [Cvetaeva], Correspondance (1922-1936), traduite du russe par Éveline Amourski \& Luba Jurgenson, Éditions des Syrtes, Coll. « Littérature étrangère », Paris, 2005, 688 p.

18. Berberova N. : Alexandre Borodine, 1834-1887, Actes Sud, coll. « Musique », Paris, 1990, 91 p. ; Le cap des tempêtes, traduit du russe par Luba Jurgenson, Actes Sud, coll. "Un endroit où aller ", Paris, 2002, 300 p.; La grande ville, traduit du russe par Luba Jurgenson, Actes sud, coll. «Un 
Endroit où Aller », 2003, 30 p.; Récits de l'exil (2 tomes) traduit du russe par Luba Jurgenson, postface de Hebey P., Actes sud, coll. « Babel », nº 62 \& 78, 2004, 365 p. +192 p.

19. Gorenstein Fr., Compagnons de route, traduit du russe par Luba Jurgenson, De Fallois / L'Âge d'Homme, Lausanne, 1988, 178 p.

20. Guirchovitch [Giršovič] L. : Apologie de la fuite, traduit du russe par Luba Jurgenson, Verdier, Coll. « Poustiaki », 2004, 400 p. ; Têtes interverties, traduit du russe par Luba Jurgenson, Verdier, Coll. « Poustiaki », 2007, 448 p.

21. Koroliov [Korolëv] A., La tête de Gogol, traduit du russe par Luba Jurgenson, Calmann-Levy, 2005, $252 \mathrm{p}$.

22. Gontcharov [Gončarov] I. A., Oblomov, traduit du russe par Luba Jurgenson, L'Âge d'Homme, coll. «Classiques slaves », Lausanne, 1990, 475 p.

\section{INDEX}

Mots-clés : émigration, exil, littérature russe, traduction littéraire

Index géographique : France, Russie, URSS

Index chronologique : communisme, époque contemporaine, post-communisme, XXe siècle, XXIe siècle

\section{AUTEURS}

\section{PETRA JAMES}

Responsable de la Chaire de tchèque, section de Langues et Littératures modernes, option Slaves, de l'Université Libre de Bruxelles (Belgique) ; membre du comité de rédaction et du comité scientifique de Slavica Bruxellensia

\section{NICOLAS LITVINE}

Université Libre de Bruxelles (Belgique) ; membre du comité de rédaction de Slavica Bruxellensia 